\title{
PRIMERA CUMBRE DE MIGRANTES DE AMERICA LATINA EXCEDE ESPECTATIVAS: MIGRANTES IDENTIFICAN LAS PRIORIDADES PARA UNA ACCIÓN COMÚN
}

OSCAR CHACÓN*

AMY SHANNON**

E n una emocional coincidencia, la Primera Cumbre de Comunidades Migrantes Latino Americanas fue inaugurada el Día de la Madre, el 10 de mayo de 2007. Calentados por el sol de primavera y por los homenajes a las madres que enfrentan la separación familiar, resultado de la aplicación de políticas migratorias injustas, cientos de líderes migrantes latinoamericanos, quienes ahora residen en países alrededor del mundo, se reunieron en Morelia, Michoacán, México para discutir una agenda propositiva al nivel hemisférico.

Esta histórica cumbre logró reunir a más de mil participantes que se dedicaron durante cuatro días al intercam- bio de ideas y experiencias que, sin lugar a dudas, enriquecerá el trabajo de las organizaciones de migrantes que fueron parte de ella. Los organizadores de la Cumbre, miembros de redes y organizaciones lidereados por migrantes en los Estados Unidos de América, Europa y América Latina se reunieron gracias a un esfuerzo compartido entre cientos de organizaciones, que fue respaldado por el apoyo generoso de los gobiernos del estado de Michoacán, el Municipio de Morelia en México y por numerosos donantes privados. La Cumbre representa la primera reunión de tantas organizaciones lidereadas por migrantes, con el fin de avanzar hacia una agenda propia.

* Director Ejecutivo de la Alianza Nacional de Comunidades Latinoamericanas y Caribeñas y miembro del Comité Internacional de la Cumbre.

** Directora Interina de Enlaces América, Secretariado de la Cumbre.

NotA DE LA AUTORA: Agradecemos la importante labor del equipo de voluntarios que sistematizaron los resultados de los talleres, así como la síntesis de las recomendaciones que fue preparada por la escritora Sonia Vasquez. Los resultados detallados de los talleres se encuentran en la página web www.cumbredemigrantes.org en español e inglés. 
¿POR QUÉ UNA CUMBRE DE COMUNIDADES MIGRANTES DE AMÉRICA LATINA?

El Continente Americano es un continente de migrantes. Prácticamente todos los países del hemisferio son impactados de una forma u otra por el fenómeno migratorio. Ya sea por ser países de emisión, recepción o tránsito de migrantes, o bien, una combinación de estas condiciones. Para algunas naciones, las remeses enviadas por sus connacionales en el exterior han llegado a representar un factor de estabilidad sumamente importante para las economías locales. En la medida en que las migraciones han crecido en las últimas dos décadas, han surgido también organizaciones de migrantes en muchos países. Estas organizaciones tienen expresiones múltiples, pero como regla general comparten la aspiración de construir comunidades saludables, tanto en el país de destino de las migraciones, como también en los países de origen.

La migración es un fenómeno global que idealmente debe abordarse desde esta óptica. Sin embargo, hay varias razones por las cuales los organizadores decidieron enfocar la Cumbre en los migrantes originarios de América Latina. En parte, el enfoque regional podría potenciar un proceso de seguimiento más concreto de los acuerdos tomados o líneas de trabajo identificadas. Además, la concentración de emigrantes de América Latina en los Estados Unidos, país donde los extranjeros de origen latinoamericano representan casi el 75\% de todos los extranjeros en ese país, permitió la participación de muchos grupos nacionales. Como factor pragmático, la predominancia del idioma español entre grupos nacionales de América Latina, facilitó también los intercambios. Sin embargo, cabe mencionar que la participación de una delegación de migrantes de origen africano, radicando actualmente en Francia, se incorporó a la Cumbre y sus aportaciones enriquecieron las deliberaciones, permitiendo una comparación entre las realidades enfrentados por las comunidades transnacionales en diferentes contextos.

\section{OBJETIVOS Y RESULTADOS}

El objetivo último de la Cumbre fue fortalecer el posicionamiento de las organizaciones de migrantes originarios de América Latina como un colectivo de protagonistas clave, en función del desarrollo de comunidades saludables en lugares de destino y de origen de las migraciones. Los espacios de diálogo y aprendizaje que se realizaron a lo largo de los cuatro días de la Cumbre reafirman la rica diversidad de opiniones que caracteriza a las comunidades de migrantes latinoamericanos, así como también a los múltiples impactos y la variedad de puntos de vista desde los que se articulan propuestas de soluciones a los desafíos y oportunidades que las migraciones engendran.

Los temas tratados durante los cuatro días de la Cumbre fueron muy variados. Durante la jornada del 10 de mayo se hizo énfasis en el rol de la mujer migrante y en los impactos sociales de la migración. Las presentacio- 
nes emotivas y muy personales de la actríz mexicana Cecilia Suárez y de Ángela Sambrano, Presidente de la

Alianza Nacional de Comunidades Latino Americanas y Caribeñas (NALACC por sus siglas en inglés) conmovieron a todos los participantes. Otros temas que fueron tocados este primer día incluyeron el liderazgo de la mujer en el fenómeno migratorio; salud y migración; pandillas y temores sociales; derechos humanos; costo humano y social, así como liderazgo juvenil.

El aspecto cultural es muy importante para las comunidades transnacionales, y no fue ignorado durante la Cumbre. Se organizó una exhibición de pinturas, fotografías, cine y una colección de dibujos infantiles titulada «Vidas sin Fronteras». Todas estas actividades fueron co-auspiciadas por el Museo Regional de Morelia. La colección de dibujos infantiles refleja los impactos de la migración en las vidas de los migrantes, sus familias y sus comunidades.

Para el 11 y 12 de mayo las mesas redondas y plenarias se enfocaron en el por qué de la migración, sus raíces en la pobreza y la globalización, entre otras. Además se profundizó en el papel que juegan las organizaciones de migrantes en la promoción del bienestar de sus lugares de origen, así como en los múltiples roles que están jugando las organizaciones transnacionales de migrantes en la incidencia a favor de reformas migratorias, organización social y procesos de integración, inversiones locales, y la participación cívica.

El intenso programa de actividades de la Cumbre concluyó el domingo 13 de mayo con la plenaria: «Co- munidades migrantes organizadas como expresión de la nueva sociedad civil transnacional: perspectivas hacia una agenda hemisférica», así como con una revisión de propuestas y una reflexión acerca de cuáles serán los próximos pasos a seguir.

En su declaración ante la última plenaria, los integrantes del Comité Organizador de la Primera Cumbre sintetizaron los siguientes aspectos de análisis y valores compartidos que fueron escuchados durante el evento:

1. Dadas las condiciones de exclusión cultural, social, política y económica que se derivan del modelo dominante de globalización económica bajo el cual hemos vivido durante el último cuarto de siglo, y su llano fracaso, urgen nuevas alternativas. El patrón estructural de empobrecimiento e injusta distribución de la riqueza es agravado por conflictos civiles, a menudo violentos, como también por desastres naturales cada vez más frecuentes. La conjugación de estos factores constituyen las causas fundamentales de los grandes flujos migratorios que acontecen en América Latina y el resto del mundo en la actualidad. Además de ser estos los elementos que causan las migraciones, la creciente desigualdad económica en las sociedades de los países de destino se ha convertido en los últimos años en un agente más de ataque contra poblaciones migrantes, en la medida en que sectores políticos anti-inmigración culpan a los extranjeros por dicho patrón de deterioro.

2. La vida de los y las migrantes se ha visto sistemáticamente amenaza- 
da y violentada como consecuencia de un esfuerzo constante por despojarnos de nuestra dignidad humana. Las campañas publicitarias y los esfuerzos de adopción de leyes que parten de la premisa de que los migrantes de hoy somos una amenaza, pretendiendo criminalizar la condición de ser indocumentados, o levantar muros fronterizos para supuestamente mantenernos fuera de su territorio, nos lleva a concluir que la lucha por conseguir que se nos reconozca plenamente como seres humanos, dotados de derechos inalienables reconocidos internacionalmente, constituye una de las empresas más importantes a seguir por parte de las organizaciones de los y las migrantes, con más ahínco y creatividad.

3. Que a consecuencia de las obsoletas e injustas leyes migratorias hoy vigentes en países de origen, tránsito y destino, las migraciones han tenido impactos sociales negativos que van desde la fragmentación involuntaria de los grupos familiares y sus múltiples implicaciones, hasta los vejámenes a la dignidad de las personas migrantes, que incluyen la muerte, en algunos casos a manos de cuerpos de seguridad y patrullas fronterizas. Lamentablemente hay un enorme vacío de políticas públicas que permitan sortear debidamente todos estos desafíos.

4. Potenciar el nivel de organización transnacional de las comunidades migrantes en sus diversas expresiones, para alcanzar cada vez más una mayor capacidad de influencia en los procesos de toma de decisio- nes que impactan nuestras vidas en todos los ámbitos, deberá seguir siendo la tarea más importante a fortalecer de manera continua. Dicha organización deberá conjugarse creativamente con una práctica de construcción de alianzas y articulación de convergencias que permitan a las comunidades inmigrantes ir avanzando en pro de nuestros intereses de manera cada vez más contundente.

\section{PASOS PRÓXIMOS}

Antes de la clausura de la Primera Cumbre de Migrantes, los participantes empezaron a insistir en la necesidad de una Segunda Cumbre. Los miembros del Comité Organizador Internacional acordaron que lo mejor sería esperar cuando menos dos o tres años para poder dar seguimiento a algunos de los acuerdos y las ideas generadas en los talleres. El Comité Organizador Internacional se comprometió a continuar sirviendo como un espacio de coordinación de las maneras concretas en que las organizaciones representadas hasta ahora en dicho comité, buscarán llevar acabo múltiples tareas de seguimiento que han quedado identificadas a lo largo de la primera Cumbre.

Además, los organizadores identificaron algunas tareas urgentes que se deben de empezar a llevar acabo en el interino antes de la próxima Cumbre:

1. Fortalecimiento de Organizaciones de Migrantes. Buena parte de las tareas que fueron propuestas en la 
Cumbre demandan de una mayor capacidad de abordar la opinión pública en los países de origen, tránsito y destino, con el fin de ir superando sistemáticamente los mitos equivocados acerca de los migrantes y el impacto de las migraciones.

2. Construcción de Alianzas. Además de avanzar en la consolidación de la capacidad propia de organización de las organizaciones de migrantes, es urgente fortalecer las relaciones con los distintos sectores políticos, económicos y sociales en los diferentes ámbitos impactados por las migraciones. En particular, es urgente realizar esta labor en las sociedades de destino, con el fin de conseguir su apoyo y solidaridad.

3. Incidencia Transnacional e Internacional. En última instancia, son los múltiples niveles y ramas de los gobiernos nacionales, impactados por las migraciones, así como los cuerpos internacionales multilaterales políticos y financieros, en donde deberán propiciarse los cambios de políticas públicas para lograr que hagan de las migraciones y sus efectos factores que fortalezcan el desarrollo humano y económico, equitativo y sustentable, en las sociedades de origen, tránsito y destino. Las organizaciones miembros del Comité Organizador Internacional se comprometieron a buscar un mayor grado de incidencia en tales espacios de toma de decisiones.

Con la celebración de esta Primera Cumbre, los migrantes latinoamerica- nos organizados en países alrededor del mundo escribieron una página inédita de la historia. Por primera vez fueron los sujetos mismos quienes articularon su propia agenda, además de dejar planteado lo imperativo de seguir fortaleciendo este espacio conjunto de deliberación, coordinación y actualización de su propia agenda. Esta Primera Cumbre ha contribuido también a fortalecer el entendimiento y el compromiso colectivo con la articulación de espacios globales de coordinación entre todas las comunidades migrantes organizadas del planeta. La Primera Cumbre de Comunidades Migrantes Latinoamericanas hizo una importante contribución a la definición práctica del concepto de ciudadanía global, el cual encuentra en las comunidades migrantes del planeta una de sus principales expresiones.

ANEXO 1.

PRINCIPALES TEMAS

Y PROPUESTAS PRESENTADAS

DURANTE LA PRIMERA CUMBRE DE COMUNI-

DADES MIGRANTES LATINOAMERICANAS MORELIA, MICHOACÁN-MAYO DE 2007

\section{Derechos humanos}

Contexto

Durante la primera Cumbre de Comunidades Migrantes Latinoamericanas, el tema de los derechos humanos de los y las migrantes fue un concepto fundamental que tuvo eco de manera transversal en múltiples reuniones de la cumbre. En líneas generales, el tema 
destacó porque los derechos humanos

son de carácter universal y aplican a cada ser humano sin distinción (sin importar dónde y cómo se encuentren e independientemente del sexo, país de origen, raza, religión, etc.).

Entre los elementos mencionados, la mayoría basados en convenios y declaraciones internacionales y regionales, cabe destacar: el derecho de no emigrar, de no estar forzado a abandonar el hogar y la familia; el derecho de los niños y niñas de vivir con sus madres y padres; el derecho de migrar.

Los derechos humanos son inalienables y la persona que cruza la frontera se los lleva consigo.

Los Estados tienen la responsabilidad de proteger, respetar y hacer valer los derechos. También tienen la obligación de proteger la seguridad y dignidad de los migrantes, solicitantes de asilo y refugiados que transiten por su territorio; de proteger los derechos laborales y garantías sociales de todos los trabajadores; y de respetar la diversidad cultural y el principio de la no discriminación.

Por otro parte se habló del Derecho humano a la paz donde se mejoren las condiciones de vida, desarrollo y seguridad de los ciudadanos migrantes, sus pueblos de origen, las comunidades migrantes y la familia (la paz es consecuencia de justicia social e igualdad).

La frontera (y en consecuencia los inmigrantes) es vista como una zona particularmente vulnerable y propensa a la violación de los derechos humanos.

\section{Derechos laborales}

Los derechos laborales son frecuentemente violados y hay una particular

vulnerabilidad en los trabajadores in-

Que exista una Comisión de Derechos Humanos exclusivamente para las fronteras.

- Instar al gobierno mexicano para que pare las violaciones de derechos humanos que se cometen en México, especialmente la criminalización de inmigrantes de otros países.

- Capacitar a los líderes comunitarios en Derecho Constitucional, Derechos Humanos, Derecho Internacional y sus instrumentos.

- Cooperar entre diferentes entes para incidir por los derechos de inmigrantes: sindicatos, ONGS, organizaciones de derechos de inmigrantes, etc.

- Crear redes de acción para evitar actos de injusticia social que violen los Derechos Humanos.

- Luchar contra la criminalización de personas cuyos crímenes son la asistencia al migrante en cualquier país.

- Reconocimiento y expansión de los programas para refugiados a través de nuestro continente.

- Instar a los gobiernos para que reconozcan los derechos humanos como principio fundamental para la construcción de las políticas migratorias en las Américas. 
migrantes (deficiencia en la prestación de servicios médicos, redadas, salarios, condiciones de trabajo, etc.). Existe la necesidad de cooperación entre diferentes movimientos para incidir por los derechos de inmigrantes-sindicatos, ONGS, organizaciones de derechos de inmigrantes, etc. No se puede tratar con migración laboral si no se aborda la gran brecha de salarios entre países que se están desarrollando y los que ya están más avanzados. La lucha por derechos laborales requiere un enfoque en los derechos de trabajadores a ambos lados de la frontera, tiene que tener una perspectiva internacional y/o global.

PropuestaS DE ACCIÓN

- Capacitar al migrante sobre sus derechos laborales (talleres de información y capacitación preventiva sobre los derechos laborales de los trabajadores migrantes con la participación de Federaciones y Clubes).

- Utilizar más herramientas legales a nivel internacional para proteger los derechos laborales. Hay que pensar en cambios económicos que no sólo se enfoquen en situaciones nacionales, sino internacionales y globales: enfocar el trabajo en función de los trabajadores a ambos lados de la frontera (perspectiva global).

- Crear alianzas multinacionales para la defensa de los derechos humanos y laborales de los trabajadores migrantes.
- El único sindicato que va a funcionar en un mundo global es aquel que comparte valores con la comunidad. Tenemos que recordar que la historia de sindicatos en Estados Unidos es una historia de inmigrantes de Irlanda, Italia y África, por lo que hay que aprender de estas historias en nuestras propias luchas.

- El movimiento laboral tiene que responsabilizarse ante la necesidad de integrar el liderazgo Latino y de la gente inmigrante. Tenemos que enfocarnos en cómo desarrollar este liderazgo incluyendo otros grupos.

- Terminar con los intermediarios y reclutadores de migrantes, ya que esto eleva el costo y riesgos de la contratación del propio migrante.

- Tratar los pagos atrasados que el gobierno les debe a muchos migrantes que salieron de su país hacia Estados Unidos como «braceros».

\section{Cultura, medios de comunicación y migración}

Contexto

La cultura y la identidad juegan un importante papel en la vida de los migrantes y sus hijos. Se parte del concepto de que la cultura y la identidad no son conceptos fijos sino que cambian y se transforman, que van de la mano con el paso del tiempo y las dinámicas de los procesos migratorios.

Para muchos migrantes se presenta la preocupación de cómo mantener 
la cultura del país de origen en futuras generaciones. A la vez, se presentan los siguientes elementos: el sentimiento de bi-nacionalidad para los hijos de migrantes o el sentimiento de que "no soy de aquí, ni de allá»; la importancia de que los padres migrantes enseñen a sus hijos su cultura y la historia de su país; las dificultades que tienen los hijos de migrantes y los padres migrantes por el hecho de ser parte de mundos diferentes. Es fundamental el diálogo entre las generaciones de inmigrantes para conservar la cultura de origen y entender la de destino.

Por otra parte, en países como Estados Unidos, la migración es un choque cultural y los medios de comunicación contribuyen a una visión restrictiva, por lo general negativa, de la migración. La participación directa de los inmigrantes en la producción cultural (por ejemplo: las películas mostradas durante la cumbre) es un método eficaz para hablar de los temas de la migración desde el arte y la cultura.

En España es interesante que la participación de los migrantes está revolucionando el medio empresarial, los medios de comunicación (que ya hay miles en propiedad de latinos) que promueven el comercio y el turismo. Lo mismo en el caso de la Iglesia y la migración, que también es otro proceso de transformación.

\section{Propuestas DE ACCIÓN}

- Que los gobiernos de los países de destino de los inmigrantes se acti- ven en la realización de programas de educación multicultural en las escuelas.

- Que se facilite la enseñanza de los idiomas del país de destino para las poblaciones migrantes. Promoción de la integración lingüística a todos los niveles, desde la alfabetización hasta su desarrollo profesional.

- Que se desarrollen campañas educativas e informativas para sensibilizar a los pueblos receptores sobre los migrantes.

- Cambiar la opinión pública a través de los mensajes en la prensa. La estrategia tiene que involucrar a las comunidades de inmigrantes, sus aportes y sus necesidades en vez de estar a la defensiva en contra de mensajes dañinos.

- Incorporación y reconocimiento de la espiritualidad de las culturas fundadoras de nuestro continente.

- Incidir en todos los medios de comunicación: televisión, prensa, radio e internet para lograr una creciente incidencia internacional

- Impulsar mecanismos de transmisión cultural. Resaltar la importancia de la cultura de origen en los países de destino, donde resulta fundamental la conservación de la lengua. Promover el fortalecimiento de intercambios culturales especialmente destinado a los niños y jóvenes hijos de inmigrantes.

- Fomentar lo positivo de pertenecer a diferentes culturas al mismo tiempo. Las ventajas de entender dos o más idiomas, dos o más modos de pensar. En este sentido se habló mucho del tema del rescate 
de la diversidad como concepto positivo.

- Comprometer a los gobiernos de los países de origen y de destino a fortalecer los programas de educación intercultural.

\section{Desarrollo local}

y políticas de desarrollo

El desarrollo local posee las siguientes características: 1) es multidimensional: la dimensión económica es importante, pero no es la única; 2) multiactoral: hay cuatro actores infaltables: gobiernos locales, gobierno estatal, sociedad civil organizada y sector privado. Es importante desarrollar proyectos productivos que garanticen empleo e ingresos en las comunidades de los países de origen. La clave es que todos los actores del desarrollo local participen: migrantes entre sí, migrantes con las comunidades, gobierno federal, estatal, municipal y empresas.

Durante la Cumbre, hubo desacuerdos sobre el papel que los gobiernos juegan en el desarrollo local. Las remesas contribuyen al desarrollo local pero no deben de absolver al país de origen de su responsabilidad. Se mencionó que no es justo que el migrante invierta en infraestructura (agua, drenaje, electrificación...) ya que eso le corresponde al Estado (gobierno).

Como ejemplo de desarrollo local a través de migrantes se mencionó cómo Federaciones y Clubes (zacatecanos y michoacanos, por ejemplo) están llevando a cabo proyectos productivos a través del programa $3 \times 1$ (con fondos federales, estatales y municipales). En algunos casos el programa se ha convertido en $4 \times 1$ con aportaciones de compañías privadas.

Se denunció a la globalización actual porque globaliza la pobreza, pero no el desarrollo.

\section{Propuestas}

- Educar a los migrantes y las comunidades de origen sobre temas financieros.

- Promover el uso de las remesas para la creación de empleo y proyectos productivos. Al mismo tiempo, aumentar las oportunidades para que los actores locales y migrantes creen desarrollo en las comunidades y diversificar las inversiones que se hacen con las remesas.

- Crear cultura del ahorro y estructurar alrededor del mismo la capacidad de inversión. Es necesario «bancarizar» a los miembros de las familias de migrantes.

- Exigir al gobierno la inversión en infraestructura y la generación de empleos, así como una política más activa en la defensa de los derechos humanos de los migrantes.

- Buscar mecanismos eficientes para evitar que las remesas de dinero no se pierdan en las transferencias binacionales.

\section{Remesas}

ConteXto

Las remesas constituyen dinero privado. No deben de absolver el país de origen de su responsabilidad con el desarrollo económico aunque las reme- 
sas tengan un importante papel en el desarrollo local. Asimismo, las remesas no pueden jugar el mismo papel que una buena política rural del gobierno. Otro aspecto mencionado fue el asunto de que los gobiernos han obligado a los migrantes, en los hechos, hacerse cargo de las obligaciones que son del Estado en materia de desarrollo.

Se debe exigir que el Estado cumpla con sus obligaciones de desarrollo. En algunos casos las remesas pueden ser causa de la migración. La construcción de vivienda es una forma de convertir remesas en patrimonio. Asimismo, se comentó que la política en materia de envíos de remesas la imponen las agencias y los gobiernos, cuando requiere también la participación de la Comunidad Migrante.

Un punto de discusión fue el que los grandes negocios de las empresas de envíos no tienen interés en el desarrollo de las comunidades, cobran caro y ocultan información, particularmente sobre sus ganancias. El sistema financiero lucha por las remesas de los migrantes, particularmente entre los bancos y empresas de envío de dinero.

Las leyes federales de transparencia y acceso a la información pública gubernamental facilitan la transparencia, que cualquier persona pueda solicitar información significa que todas las decisiones hechas por el gobierno tienen que ser documentadas y justificadas.

Las brechas entre aquellos que reciben remesas y los que no las reciben son más y más grandes en muchos países en América Latina.
Respecto a este tema, también se habló sobre el proceso de enviar remesas y los «controles» que existen (en algunos países, como España, es difícil para los indocumentados enviar dinero a sus países) y el impacto de dichos «controles» (las personas envían dinero a través de vías irregulares).

PROPUESTAS DE ACCIÓN

- Promover el uso de las remesas para la creación de empleo y proyectos productivos. De igual manera, se propone diversificar las inversiones que se hacen.

- Se deben redefinir las políticas públicas en el ámbito de la cooperación internacional, como la revisión de microcréditos.

- Desarrollar nuevos productos financieros que respondan a necesidades transnacionales.

- Siempre buscar la manera de bajar los costos de las transferencias.

- Evitar el control y dependencia de las mujeres a través de las remesas.

- Que gobiernos locales y estatales visiten a las comunidades de destino para rendir cuentas del dinero de las remesas de los migrantes establecidos en el país de destino.

Salud

Contexto

Es importante promover la salud física y mental en la comunidad migrante. La salud emocional se ve afectada por factores como el choque cultural, separación familiar, etc. Estos elementos llevan al individuo a la depresión y an- 
siedad, así como a la apatía y el miedo a buscar ayuda psicológica o médica. Además hay que tomar en cuenta el acceso a la salud e información/ educación relacionada al tema, en la comunidad migrante.

PRopuestas

- Reafirmar que la salud es un derecho universal.

- En las políticas y convenciones sobre migración incluir el tema de la salud física y mental. Hay que subrayar a la salud como eje integral bajo la consigna y objetivo de crear «Comunidades Migrantes Saludables», lo cual involucra lo social, económico, cultural, etc., especialmente en el creciente tema de las drogas.

- Trabajar con organizaciones y medios de comunicación social para informar y orientar a la comunidad sobre temas como: uso de medicina tradicional combinada con la medicina occidental; uso de medicina y terapia ocupacional, el sida y la sexualidad responsable.

Además, apoyo a las familias para abordar estos temas con sus hijos.

- Nombrar un día nacional de concientización y sensibilización con campañas de prevención sobre la salud física y mental.

- Exigir que los países receptores de migrantes ratifiquen, firmen y pongan en práctica la Convención Internacional sobre Migrantes Trabajadores y sus Familias.

- Combatir la contaminación que perjudica la salud de los trabajadores en el exterior.
- Promover el turismo ecológico, biotecnología y programas ecologistas de acción para evitar la contaminación y el desgaste del suelo con insecticidas químicamente industrializados, previniendo, así, problemas de salud y mejorando, con ello, las condiciones de vida.

- Que el tema de la salud se revise y se tome en cuenta por parte de los legisladores.

\section{Organización y \\ participación cívica}

ConteXto

El tema de la organización de los migrantes es fundamental. Los migrantes son entes transnacionales que abogan por sus derechos en el país de origen y en el de destino. Es importante que el migrante se involucre en la vida política del país de destino y origen, conozca sus derechos y los exija. Hay que incidir en ambos países (origen y destino). El derecho al voto (y su ejercicio) se vuelve fundamental en tal sentido y fue mencionado en múltiples talleres y mesas de la Cumbre. Por otra parte, se presenta un gran desafío al tratar de involucrar a la gente en la participación cívica.

Lo que hacen los grupos/ federaciones/clubes varía dependiendo del grupo. Estas organizaciones buscan incidencia política a través de contacto con grupos de poder (directivos, oficiales electos, etc.), pacs (comités de acción política), foros, trabajo con los gobiernos de los países de origen, marchas y eventos para la reforma migratoria. 
Por otra parte, la binacionalidad trae preguntas sobre la redefinición de estado, ciudadanía flexible, desterritorializacion, comunidades transnacionales, migración, fronteras, nuevas formas de membresía política, soberanía, lealtad y patriotismo y derechos humanos.

\section{Propuestas}

- Capacitar a los líderes migrantes en diversos temas incluyendo los instrumentos de derecho internacional; las instituciones multi-laterales; el funcionamiento de los sistemas políticos de sus países de origen y de destino.

- Educar sobre la importancia del involucramiento social, político y económico de las comunidades migrantes en países de origen y destino.

- No sólo se debe enfocar en los inmigrantes para hacer un cambio positivo. Se debe organizar a los que quedan en el país de origen para cambiar la situación. Capacitación de familiares de migrantes en los países de origen, con el fin de fortalecer el vínculo con sus contrapartes en los países de destino.

- Que los inmigrantes mantengan un movimiento social y también que establezcan la institucionalización de organizaciones inmigrantes que puedan ser conocidas a nivel internacional. Ante el proceso de globalización es preciso trazar planes y plantear estrategias que tomen en cuenta a las comunidades de migrantes. La perspectiva: que dichas comunidades sean propulsoras del trabajo comunitario para formular estrategias y acciones conjuntas comunes.

- No se puede tomar acción sin infraestructura, es necesario crear organización política, social y económica.

- Hacer trabajo ideológico para concientizar sobre los aportes y el valor que hay en la inmigración (acabar o disminuir la retórica antiemigrante).

- Compartir experiencias: aprender de los modelos organizativos y esfuerzos de las comunidades migrantes alrededor del mundo y generar estrategias para fortalecer esfuerzos y establecer alianzas con otros grupos de inmigrantes. A la vez, hay una necesidad de fortalecer la base misma de cada pueblo (Por ejemplo, mexicanos en Chicago, etc.).

- Seguir trabajando por el derecho del voto en el extranjero.

- Crear iniciativas de ciudadanía y específicamente promover la ciudadanía dual.

- Que la reforma migratoria en discusión en Estados Unidos adopte a la familia como su eje central y universal.

- Articular el movimiento migrante con otras luchas sociales y otros movimientos progresistas de la sociedad civil, como los de género, indígenas y muchos otros más.

- Buscar la autogestión de recursos para tener autonomía de decisión, hacer una actividad sustentable en el largo plazo y evitar contradicciones y conflictos.

- Aprovechar la agenda de grandes instancias internacionales para intervenir en ellas unidos. 
Asuntos de género

$y$ liderazgo femenino

Contexto

La migración femenina va en aumento, se hizo un análisis sobre las razones de este crecimiento. Entre los puntos abordados estuvo la importancia de crear redes y organizaciones de apoyo a mujeres migrantes (grupos de autoayuda, mecanismos de entrenamientos y educación), integración de la mujer al país de destino e incremento de su participación.

El rol del hombre y la mujer en las migraciones y otros ámbitos de la vida cotidiana ha sufrido transformaciones a lo largo de la historia. Hay una tendencia de la mujer por luchar para obtener una mayor equidad de género y una mayor participación en la vida cívica en general. Ha aumentado su independencia y también sus responsabilidades.

PROPUESTAS DE ACCIÓN

- Aumentar la participación activa de la mujer en las redes migrantes. Formar una Red Internacional de Mujeres Migrantes que no anule las redes y organizaciones ya existentes. Fortalecer el movimiento asociativo inmigrante de las mujeres, creando desarrollo económico y promoviendo incidencia política.

- Transnacionalizar el trabajo de la mujer migrante. Dar visibilidad y reivindicar la incidencia económica del trabajo de las mujeres y luchar contra la segregación ocupacional. Velar por el respeto y fiel cumplimiento de los derechos la- borales de las mujeres inmigrantes trabajadoras en los diferentes sectores.

- Dar a conocer la influencia de la migración femenina en los cambios sociales, económicos, culturales y políticos de los países.

- Tratar de lograr una equidad de género en las organizaciones transnacionales.

- Facilitar cambios a nivel estructural, llamando la atención a la transversalización de la realidad de género a todos los niveles de la experiencia migratoria. Revisar las leyes migratorias y reivindicar la inclusión de la perspectiva de género.

- Estudiar la migración por causas de género, violencia contra la mujer, violencia intrafamiliar, prácticas culturales contra la mujer y por represión sexual. Crear iniciativas para tratar la violencia de género a partir de las relaciones de pareja, contemplando esta realidad desde los más jóvenes. Procesos de educación hacia la equidad de las mujeres para evitar la violencia de género.

- Desterrar la trata de las mujeres y niños y violación de sus derechos humanos. Contemplar el protocolo de las Naciones Unidas relativo a la criminalidad transnacional organizada, sobre la trata de los seres humanos, especialmente la trata de mujeres para la prostitución.

- Evitar el control y dependencia de las mujeres a través de las remesas.

- Procesos legales: facilitar la obtención de documentos del colectivo migrante en general, y de las mujeres en particular para asegurar la mayor protección de la mujer en la

2007 SEGUNDO SEMESTRE MIGRACIÓN Y DESARROLLO 
sociedad de origen y de destino, así como en los países de tránsito.

- Tratar el tema de la migración femenina en relación con las redes familiares, especialmente de jóvenes y ancianos. Buscar la igualdad de derechos haciendo énfasis en los procesos de educación de los jóvenes en varios niveles.

- Facilitar el diálogo entre mujeres de diversos estratos sociales y económicos. Asegurar la inclusión de las contribuciones de las mujeres en situación económica más precaria y por lo tanto con menor "Voz» en el campo político.

- Crear campañas de sensibilización y trabajo educativo, tratando el género no sólo como una realidad de «mujeres», sino como una realidad compartida por hombres y mujeres, que permea día a día y que afecta a la sociedad en su totalidad. talleres se mencionaron, como parte de su realidad cotidiana, los siguientes aspectos: la persecución, la violencia, la exclusión (no tienen acceso a lugares deportivos y programas de entrenamiento entre otros), la falta de oportunidades y de orientación que traen como consecuencia falta de metas en los jóvenes, quienes finalmente se desconectan del sistema. Elementos todos que refuerzan la falta de comunicación con los adultos, el gobierno y entre ellos mismos.

Por otra parte, se mencionó la desconexión del mundo formal de los adultos; el gobierno se desentiende de los jóvenes y la publicidad condiciona su conducta. Los adultos no trabajan conjuntamente con los jóvenes. No es la migración lo que produce la delincuencia, sino que ésta se crea por la violencia, el sistema injusto, las políticas de estado.

\section{Liderazgo juvenil}

Los jóvenes perciben la necesidad de sumar esfuerzos y compartir experiencias positivas y negativas, así como de establecer proyectos de inclusión de los jóvenes, desde los propios jóvenes y desde su perspectiva. Es importante crear y fortalecer redes de servicio y ayuda a jóvenes (prevenir la violencia y no reprimirla). Hay que trabajar para todos, para generar el cambio de todos (en relación con la juventud y la migración).

Existe una tendencia de ver a los jóvenes como criminales. Durante los
- Plantear estrategias haciendo a los jóvenes partícipes de los cambios, dándoles poder y responsabilidad. Los jóvenes son sujetos de acción, con derechos y obligaciones. Es importante trabajar junto a ellos y no delante de ellos.

- Incentivar que los jóvenes tengan perspectiva de su propia vida en lugar de crear estereotipos.

- El problema de las pandillas se tiene que ver desde la óptica de la organización comunitaria. Para resolverlo, es mejor prevenir y no reprimir. 
- Presionar por cambios en las leyes migratorias para que permitan obtener un estatus legal a una persona aunque se haya delinquido.

- Impulsar mecanismos de transmisión cultural. La importancia de la cultura de origen en los países de destino, la conservación de la lengua de los padres.

- Promocionar el fortalecimiento de intercambios culturales especialmente destinado a los niños y jóvenes hijos de inmigrantes.

- Creación de proyectos de orientación para jóvenes en los países de destino para facilitar el proceso de integración en el tejido social educativo.

- Realizar talleres que contemplen las realidades de migrantes jóvenes en varias regiones (Europa, Estados Unidos y América Latina) así como la promoción de encuentros regionales de jóvenes.

\section{Políticas públicas}

y legislación

CONTEXTO

En el plano legislativo se discutieron varios temas y se destacó particularmente el caso de Estados Unidos, su frontera con México y las políticas migratorias (o la carencia de ellas) en ambos países. Entre los puntos mencionados se encuentran: afinar mecanismos de diálogo con otros gobiernos; combatir efectos perniciosos de una reforma migratoria en Estados Unidos; llevar a cabo programas coordinados entre diferentes instancias de gobierno para combatir tráfico de personas, la inseguridad y la violen- cia en las fronteras; garantizar los derechos humanos de los migrantes, sobre todo de los grupos vulnerables (despenalizar migración indocumentada); actualizar la Ley General de Población en materia migratoria y adecuarlo a los ordenamientos de la Convención Internacional para la Protección del Trabajador Migrante y sus familias.

Al mismo tiempo, se dijo que el tratamiento inadecuado del problema migratorio provoca problemas de narcotráfico, delincuencia organizada, trata de blancas, prostitución, pederastia y pornografía infantil entre otros. Se hizo mención de que la comunidad latina en Estados Unidos no tiene voz en la política exterior de este país hacia América Latina y eso se necesita cambiar pues es necesario que el enfoque del tema migratorio recupere la dimensión humana, pero con la voz migrante.

\section{Propuestas}

- Impulsar leyes justas y reformas a las leyes migratorias que reconozcan la contribución de los migrantes a la sociedad tanto en países de origen como de destino.

- Reformar leyes que criminalicen al inmigrante.

- Reforzar la «Red de Legisladores Latinoamericanos por la Migración", a partir del encuentro de legisladores durante la Cumbre de Morelia y la próxima reunión en Los Ángeles, Estados Unidos.

- Unificar una Declaración de denuncia de los migrantes latinoamericanos y otros en Estados Uni- 
dos contra los intentos legislativos anti-inmigrantes en ese país para ser entregada al Secretario General de la ONU. Dada la relevancia simbólica y el impacto ejemplar de lo que ocurra con dicha legislación, hacer lo mismo en relación con la Unión Europea.

- Rechazo a los muros por ser una política contraria a la integración. Los muros físicos, intelectuales e ideológicos limitan y obstaculizan la participación del migrante en la sociedad civil.

- Exigir a Estados Unidos parar la militarización de la frontera. De igual manera, exigir a México desmilitarizar la frontera con Guatemala.

- Es urgente desmantelar las redes de traficantes de migrantes.
- Tejer una red internacional para hacer políticas públicas de, por y para los inmigrantes y proponer políticas transnacionales por sus derechos.

- Promover políticas públicas en los países de origen que generen desarrollo para evitar la migración.

- Que las organizaciones se coordinen con los Consulados con metas concretas a corto, mediano y largo plazo.

- La ratificación y firma de convenios y tratados internacionales en defensa de los trabajadores, los niños, las mujeres y los migrantes.

- Exigir a los Estados respeto a políticas públicas y legislación sobre la migración, sobre los servicios básicos como salud y educación y el trato integral, no meramente económico, del fenómeno migratorio. 